\title{
ASYMPTOTICALLY AUTONOMOUS MULTIVALUED DIFFERENTIAL EQUATIONS $\left({ }^{1}\right)$
}

\author{
BY
}

JAMES P. FOTI

\begin{abstract}
The asymptotic behavior of solutions of the perturbed autonomous multivalued differential equation $x^{\prime} \in F(x)+G(t, x)$ is examined in relation to the behavior of solutions of the autonomous equation $x^{\prime} \in F(x)$ assuming that all solutions of the latter approach zero as $t$ approaches $\infty$.
\end{abstract}

For multivalued functions $F$ and $G$ whose values are nonempty subsets of $d$-dimensional Euclidean space, $R^{d}$, the generalized differential equation

$$
x^{\prime} \in F(x)+G(t, x)
$$

is said to be asymptotically autonomous if $G(t, x)$ becomes small in some sense as $t \rightarrow \infty$. The main result of this investigation establishes the relationship of the asymptotic behavior of solutions of (1) to that of solutions of the autonomous equation

$$
x^{\prime} \in F(x)
$$

THEOREM 1. Let $F$ be a positive-homogeneous upper semicontinuous mapping from $R^{d}$ (d-dimensional Euclidean space) to the nonempty, compact, convex subsets of $R^{d}$ such that all solutions of (2) approach zero as $t \rightarrow \infty$. Let $G$ be a mapping from $R^{1+d}$ to the nonempty subsets of $R^{d}$ such that $G(t, \cdot) \rightarrow 0$ as $t \rightarrow \infty$ uniformly on nonempty compact subsets of $R^{d}$. If $\phi$ is a bounded solution of $(1)$ on $[0, \infty)$ then $\phi(t) \rightarrow 0$ as $t \rightarrow \infty$.

If $F$ and $G$ are single-valued functions, denoted by $f$ and $g$, respectively, the equations (1) and (2) are ordinary differential equations and the asymptotic behavior of the solutions is discussed, for example, by Strauss and Yorke. One of their results [7, p. 180] guarantees that all (classical) solutions of

$$
x^{\prime}=f(x)+g(t, x)
$$

which are bounded on $\left[t_{0}, \infty\right)$ tend to zero as $t \rightarrow \infty$ provided that $f$ and $g$ are

Presented to the Society, January 25, 1975; received by the editors February 11, 1975. AMS (MOS) subject classifications (1970). Primary 34D05, 34D10; Secondary 34E10.

(1) This article was derived from the author's doctoral dissertation submitted to the University of Maryland. 
continuous vector-valued functions, that all solutions of the unperturbed autonomous equation approach zero as $t \rightarrow \infty$, and that $g(t, x)$ "mostly approaches zero". The last condition, which is defined in $[7, p .176]$ is satisfied, if for example, $g(t, \cdot)$ approaches zero as $t \rightarrow \infty$ uniformly on compact subsets of $R^{d}$. Other treatments of asymptotically autonomous ordinary differential equations may be found in [1]-[4] and [6]-[10].

A perturbation-type result for generalized differential equations was developed by Lasota and Strauss [5, p. 169] as an aid in their investigation of autonomous ordinary differential equations. This result, tailored to suit the present context, is presented below.

LEMMA 2. Let $F$ be a positive-homogeneous upper semicontinuous mapping from $R^{d}$ to the nonempty, compact convex subsets of $R^{d}$ such that every solution of (2) approaches zero as $t \rightarrow \infty$. Then there exist $\epsilon>0$ and $K>1$ such that for $t_{0}>0$ and $x_{0} \in R^{d}$ each solution of

$$
x^{\prime} \in F(x)+\epsilon B(|x|), \quad x\left(t_{0}\right)=x_{0}
$$

can be continued to $+\infty$ and satisfies

$$
|x(t)| \leqslant K\left|x_{0}\right| \exp \left(-\epsilon\left(t-t_{0}\right)\right)
$$

for all $t \geqslant t_{0}$.

A solution of (1) is an absolutely continuous $d$-vector valued function which satisfies (1) almost everywhere on some nondegenerate interval. For $\epsilon>0$, $x \in R^{n}$, and $A \subset R^{n}$ denote the Euclidean norm of $x$ by $|x|$ and the norm of $A$ by $\|A\|=\sup \{|x|: x \in A\}$. The distance from $x$ to $A$ is defined by $d(x, A)=$ $\inf \{|x-y|: y \in A\}$ and the $\epsilon$-neighborhood of $A$ is the set $N(A, \epsilon)=\left\{y \in R^{n}\right.$ : $d(y, A)<\epsilon\}$. The closed-origin-centered ball of radius $\epsilon$ is denoted by $B(\epsilon)$.

The multivalued mapping $H$ from $R^{n}$ to the nonempty compact subsets of $R^{d}$ is said to be upper semicontinuous if to each $\epsilon>0$ and $x \in R^{n}$ there corresponds $\delta>0$ such that $H(y) \subset N(H(x), \epsilon)$ provided $|x-y|<\delta$. The setvalued mapping $H$ defined on $R^{n}$ is said to be positive-homogeneous if $H(r x)=$ $r H(x)=\{r z: z \in H(x)\}$ for all $x \in R^{n}$ and $r>0$. The statement $H(t) \rightarrow \infty$ means that to each $\epsilon>0$ there corresponds $T>0$ such that $H(t) \subset B(\epsilon)$ for all $t \geqslant T$; that is, $\|H(t)\| \rightarrow 0$ as $t \rightarrow \infty$.

A variation of Theorem 1 , in which the perturbation term depends only on $t$, provides an approach to the proof of the main result.

THEOREM 3. Let $F$ and $G$ satisfy the hypotheses of Theorem 1 and in addition assume that $G$ is independent of $x$. Then all solutions (not just the bounded solutions) of 


$$
x^{\prime} \in F(x)+G(t)
$$

on $[0, \infty)$ approach zero as $t \rightarrow \infty$.

The proof of this theorem is based on the observation that if $\phi$ is a solution of (6) for which $G(t) \subset \epsilon B(|\phi(t)|)$ for all $t \geqslant 0$ then $\phi(t) \rightarrow 0$ as $t \rightarrow \infty$ according to Lemma 2; whereas, if $G(t) \not \subset \epsilon B(|\phi(t)|)$ for all $t \geqslant 0$ then $\epsilon B(|\phi(t)|) \subset$ $B(\|G(t)\|)$, and $\phi(t) \rightarrow 0$ since $\|G(t)\| \rightarrow 0$ as $t \rightarrow \infty$.

Proof of Theorem 3. Let $\epsilon$ and $K$ be as in Lemma 2 and let $\phi$ be a solution of $(6)$ at least on $[0, \infty)$. Define the sets $I$ and $J$ by

$$
I=\{t \geqslant 0: G(t) \subset \epsilon B(|\phi(t)|)\}
$$

and

$$
J=\{t \geqslant 0: G(t) \not \subset \epsilon B(|\phi(t)|)\} ;
$$

clearly $I \cup J=\{t \geqslant 0\}$ and $I \cap J=0$. In the light of the previous remarks, it remains to be shown that $\phi(t) \rightarrow 0$ as $t \rightarrow \infty$ when both $I$ and $J$ are unbounded sets. Since the solution approaches zero on unbounded increasing sequences from $J$, it suffices to show that $\phi$ approaches zero along an arbitrary unbounded increasing sequence from $I ;$ let $\left\{t_{k}\right\}$ be such a sequence. For $k=1,2,3, \ldots$, let $I_{k}$ denote the component (maximal connected subset) of $I$ which contains $t_{k}$ and let $d_{k}$ denote the length of this component. Let $s_{k}=\inf \left\{t \in I_{k}\right\}$ and assume, without loss of generality, that $s_{1}>1$; clearly, $s_{k} \uparrow \infty$ as $k \rightarrow \infty$. The continuity of $\phi$ provides for each positive integer $k$ a corresponding $\delta_{k}<1$ such that

$$
\left|\phi\left(s_{k}\right)-\phi(t)\right|<1 /(2 k) \text { for }\left|t-s_{k}\right|<\delta_{k} ;
$$

in addition, if $d_{k}>0$, choose $\delta_{k}<d_{k}$. Choose auxiliary sequences $\left\{\tau_{k}\right\} \subset J$ and $\left\{\tau_{k}^{*}\right\} \subset I$ such that $s_{k}-\delta_{k} \leqslant \tau_{k} \leqslant s_{k}$ and $s_{k} \leqslant \tau_{k}^{*} \leqslant s_{k}+\delta_{k}$ for each positive integer $k$; these selections guarantee that

$$
\left|\phi\left(\tau_{k}\right)-\phi\left(s_{k}\right)\right|<1 /(2 k)
$$

and

$$
\left|\phi\left(\tau_{k}^{*}\right)-\phi\left(s_{k}\right)\right|<1 /(2 k)
$$

Consequently, for $t \in I_{k}, \phi$ satisfies

$$
|\phi(t)| \leqslant \begin{cases}\left|\phi\left(s_{k}\right)\right|+1 /(2 k) & \text { for } t \leqslant \tau_{k}^{*}, \\ K\left|\phi\left(\tau_{k}^{*}\right)\right| \exp \left(-\epsilon\left(t-\tau_{k}^{*}\right)\right) & \text { for } t \geqslant \tau_{k}^{*} .\end{cases}
$$

The estimate in (12) follows from (9) and the choice of $\tau_{k}^{*}$; whereas, the estimate in (13) follows from Lemma 2. These estimates can be modified by 
(10) and (11) to obtain

$$
|\phi(t)| \leqslant K\left(\left|\phi\left(\tau_{k}\right)\right|+1 / k\right) \quad \text { for } t \in I_{k} .
$$

In particular, since $t_{k} \in I_{k}$ and $\tau_{k} \in J$, it follows that $\phi\left(\tau_{k}\right) \rightarrow 0$ as $k \rightarrow$ $\infty$ which forces $\phi\left(t_{k}\right) \longrightarrow 0$ as $k \rightarrow \infty$; thus $\phi(t) \rightarrow 0$ as $t \rightarrow \infty$, which concludes the proof.

The proof of Theorem 1 follows almost as an immediate consequence of Theorem 3.

Proof of Theorem 1. Let $\phi$ be a bounded solution of (1) which is defined at least on $[0, \infty)$, and let $C$ denote a compact subset of $R^{d}$ which contains $\phi(t)$ for all $t \geqslant 0$. Define the multivalued function $H$ by

$$
H(t)=\{y \in G(t, x): x \in C\} .
$$

Clearly, $H(t) \rightarrow 0$ as $t \rightarrow \infty$ and $\phi$ is a solution of

$$
x^{\prime} \in F(x)+G(t, x) \subset F(x)+H(t) .
$$

An application of Theorem 3 yields the desired results.

\section{REFERENCES}

1. H. A. Antosiewicz, Stable systems of differential equations with integrable perturbation term, J. London Math. Soc. 31 (1956), 208-212. MR 18, 42.

2. Fred Brauer and Aaron Strauss, Perturbations of nonlinear systems of differential equations. III, J. Math. Anal. Appl. 31 (1970), 37-48. MR 41 \#3919.

3. L. Cesari, Asymptotic behavior and stability problems in ordinary differential equations, Ergebnisse der Math. und ihrer Grenzgebiete, N. F., Band 16, Academic Press, New York; Springer-Verlag, Berlin, 1963. MR 27 \#1661.

4. E. A. Coddington and N. Levinson, Theory of ordinary differential equations, McGraw-Hill, New York, 1955. MR 16, 1022.

5. A. Lasota and Aaron Strauss, Asymptotic behavior for differential equations which cannot be locally linearized, J. Differential Equations 10 (1971), 152-172. MR 43 \#3570.

6. S. Lefschetz, Differential equations: Geometric theory, 2nd ed., Pure and Appl. Math., vol. 6, Interscience, New York, 1963. MR 27 \#3864.

7. Aaron Strauss and James A. Yorke, On asymptotically autonomous differential equations, Math. Systems Theory 1 (1967), 175-182. MR 35 \#4524.

8. - Perturbation theorems for ordinary differential equations, J. Differential Equations 3 (1967), 15-30. MR 34 \#3029.

9. - Perturbing uniform asymptotically stable nonlinear systems, J. Differential Equations 6 (1969), 452-483. MR 40 \#5998.

10. T. Yoshizawa, Stability theory by Lyapunov's second method, Publ. Math. Soc. Japan, no. 9, Math. Soc. Japan, Tokyo, 1966. MR 34 \#7896.

DEPARTMENT OF MATHEMATICS, UNITED STATES NAVAL ACADEMY, ANNAP. OLIS, MARYLAND 21402 